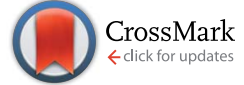

Cite this: RSC Adv., 2015, 5, 1937

Received 23rd September 2014 Accepted 1st December 2014

DOI: $10.1039 / c 4 r a 11051 j$

www.rsc.org/advances

\section{Gold nanoparticle-doped silk film as biocompatible SERS substrate $\uparrow$}

\begin{abstract}
Chengchen Guo, Genevieve N. Hall, John B. Addison and Jeffery L. Yarger*
In this work, we present a novel rapid method for fabricating biocompatible, biodegradable gold nanoparticle-embedded silk films (AuNP-silk films) that have potential applications in bioengineering and biomedical research. Thin AuNP-silk films are prepared from a AuNP-silk nanocomposite and characterized by scanning electron microscopy (SEM), solid-state nuclear magnetic resonance (NMR) spectroscopy, and UV-visible spectroscopy. Results indicate that the size and distribution of AuNPs can be well controlled in AuNP-silk films, which may have great significance in developing novel biosensors. As a proof of principle, we further demonstrate that the prepared AuNP-silk films can serve as an excellent substrate for surface-enhanced Raman scattering in trace analysis. Moreover, the solid-state NMR results show that the AuNP-silk films possess a structure similar to natural silk, indicating that the fabrication process has little effect on silk protein. Surface plasmon absorption study of AuNPs shows that the optical properties of AuNPs vary when embedded into silk films because of the higher refractive index of silk, which is demonstrated by theoretical simulation.
\end{abstract}

\section{Introduction}

Gold nanoparticles (AuNPs) have been studied for decades since they have many applications in diagnostics, therapeutics, optical sensing, and catalysis. ${ }^{1-3}$ They have fascinating optical properties derived from their localized surface plasmon resonances, which make them excellent candidates for the development of optical sensors. Of all the sensing techniques, surface-enhanced Raman scattering (SERS) based sensors are among the most well-known and popular sensors in recent research. ${ }^{4,5}$

Silks are generally defined as protein polymers that are produced naturally by some Lepidoptera larvae such as silkworms, spiders, scorpions, and flies. Silkworm silk and spider silk are the most studied and widely used and are excellent materials for biomedical applications such as drug delivery and tissue engineering because of their biocompatibility, biodegradability, and extraordinary mechanical properties..$^{6-12}$ Spider silks are known as the strongest and toughest natural fibres, possessing unrivalled extensibility and high tensile strength. ${ }^{11}$ Silkworm silk has been used as suture material for centuries

Department of Chemistry and Biochemistry, Magnetic Resonance Research Center, Arizona State University, Tempe, Arizona, 85287-1604, USA.E-mail: jyarger@gmail. com

$\dagger$ Electronic supplementary information (ESI) available: Theory of surface plasmon resonance simulation for AuNPs, chemical shifts assignments for solid-state NMR spectra, summary of experimental and simulated absorption spectra of AuNPs, comparison of experimental and simulated absorption spectra of AuNPs of various sizes in aqueous solution, and vibrational assignments obtained from Raman spectra. See DOI: 10.1039/c4ra11051j and recently has gained considerable attention as a biomaterial with various medical applications due to its slow rate of degradation in vivo and its ability to be fabricated into multiple types of materials such as fibres, films, gels, and foams. ${ }^{9}$ To extend the applications of silk materials, researchers have recently transitioned from focusing on pure silk materials to silk-based nanocomposites. Many nanocomposite materials have been fabricated by combining inorganic materials such as gold, silver, silica, graphene, and carbon nanotubes with silks. $^{12-22}$ Generally, these nanocomposite materials are prepared by in situ synthesis of inorganic nanomaterials or by employing layer-by-layer approaches. Some of the resultant properties of these composite structures may be exploited to develop novel biocompatible devices.

Surface-enhanced Raman scattering (SERS) spectroscopy is a solid technique that is used in many areas, such as solidstate physics, material science, and chemistry., ${ }^{4,523-25}$ It was first discovered by Fleischmann et al. in 1974 by observing the strong Raman signal of pyridine molecules on the roughened surface of a Ag electrode. ${ }^{26}$ In 1977, Van Duyne et al. reported that the roughened $\mathrm{Ag}$ surface could enhance the Raman signal of pyridine $10^{6}$ times. ${ }^{27}$ This unusual enhancement quickly attracted the attention of many researchers since it could be used to realize high-resolution detection, even of single molecules, and makes SERS an ideal analytical tool for the investigation of single living cells and trace analysis. ${ }^{28,29}$

In this report, we provide a novel method for making gold nanoparticle-embedded silk nanocomposites that are further fabricated into films and used as SERS substrates. The method 
is efficient and the prepared thin films are of high quality with good dispersion of gold nanoparticles of a narrow size distribution. Furthermore, the silk proteins in the films possess similar structures to those of natural silk, indicating that the properties of the silk proteins are not changed dramatically by the fabrication process. Embedding gold nanoparticles makes the films suitable for a variety of applications, including biosensors. Here we present the potential for these films to be used as a SERS substrate for trace analysis.

\section{Experimental}

\section{Chemicals and materials}

Chloroauric acid $\left(\mathrm{HAuCl}_{4} \cdot 3 \mathrm{H}_{2} \mathrm{O}\right)$, trisodium citrate $\left(\mathrm{Na}_{3} \mathrm{C}_{6} \mathrm{H}_{5}\right.$ $\left.\mathrm{O}_{7} \cdot 2 \mathrm{H}_{2} \mathrm{O}\right)$, trifluoroacetic acid $\left(\mathrm{CF}_{3} \mathrm{COOH}\right)$, 4-(dimethylamino) pyridine (4-DMAP), and rhodamine $6 \mathrm{G}$ were purchased from Sigma-Aldrich Inc. and used as received. The silkworm silks were obtained from lab-cultivated Bombyx mori silkworms. Briefly, the cocoons were first cut into pieces and then boiled in $0.02 \mathrm{M} \mathrm{Na}_{2} \mathrm{CO}_{3}$ solution for one hour to degum the silk. ${ }^{9}$ The degummed silk was washed with DI water and then dried completely under vacuum.

\section{Synthesis of citrate-capped gold nanoparticles}

Citrate-capped gold nanoparticles (AuNPs) were synthesized by a slightly modified Turkevich method..$^{30,31} 13.4 \mathrm{mg}$ chloroauric acid $(0.34 \mathrm{mM})$ was first dissolved in $100 \mathrm{~mL}$ DI water and the solution was heated to the refluxing point while being stirred. A specific amount $(0.8 \mathrm{~mL}, 1.0 \mathrm{~mL}, 1.2 \mathrm{~mL}$, or $1.4 \mathrm{~mL})$ of sodium citrate $(1 \% \mathrm{w} / \mathrm{w})$ was rapidly added to the boiling solution, which was then boiled for another $30 \mathrm{~min}$ before being cooled to room temperature. During the $30 \mathrm{~min}$ boiling period, the colour of the solution quickly changed to black and then gradually lightened to red or purple, depending on the amount of citrate.

\section{Preparation of AuNP-silk films}

$10.0 \mathrm{mg}$ dry degummed silk was dissolved into $100 \mu \mathrm{L} \mathrm{99.9 \%}$ trifluoroacetic acid (TFA) and the resultant solution was quickly injected into $50 \mathrm{~mL}$ aqueous AuNP solution under mild stirring, producing a homogenous solution. To reduce the aggregation time of the AuNP-silk nanocomposite, the $\mathrm{pH}$ was adjusted to neutral. The homogeneous solution was then stirred for about three hours until the AuNP-silk nanocomposite had completely precipitated. The nanocomposite was separated by filtration, washed with DI water and methanol, and dried overnight under vacuum. To prepare AuNP-silk films, a TFA/nanocomposite solution $(4 \% \mathrm{w} / \mathrm{w})$ was first made by dissolving $6.0 \mathrm{mg}$ nanocomposite in $100 \mu \mathrm{L}$ TFA. The films were spin-coated on precleaned glass slides with a spinning speed of $2400 \mathrm{rpm}$ $\min ^{-1}$, and subsequently washed with water and methanol. The prepared films were dried in air overnight. Bare silk films were also prepared in this manner and were used as a reference in the SERS experiments.

\section{Characterization}

The size and morphology of the AuNPs were characterized using a Philips CM200-FEG high-resolution transmission electron microscope (TEM) operating at a bias voltage of $200 \mathrm{kV}$. The TEM samples were prepared by dipping carbon-coated copper grids in the AuNP solutions and then drying them in air for over two hours. The size and distribution of AuNPs in the AuNP-silk films were determined using a Nova2000 high-resolution scanning electron microscope (SEM) operating at a voltage of $20 \mathrm{kV}$. UV-vis spectra of the AuNP solutions and the AuNP-silk films were collected with a SpectroVis Plus spectrophotometer. Solidstate NMR experiments were performed on a Bruker AVIII $400 \mathrm{MHz}$ spectrometer equipped with a $4.0 \mathrm{~mm}$ double resonance probe operating in double resonance $\left({ }^{1} \mathrm{H} /{ }^{13} \mathrm{C}\right)$ mode at a spinning speed of $10 \mathrm{kHz} .{ }^{1} \mathrm{H} \rightarrow{ }^{13} \mathrm{C}$ cross-polarization magicangle-spinning (CP-MAS) NMR experiments were performed to determine the structure of the samples. ${ }^{32,33}$ The CP condition of ${ }^{1} \mathrm{H} \rightarrow{ }^{13} \mathrm{C}$ CP-MAS NMR experiments consisted of a $4.5 \mu \mathrm{s}{ }^{1} \mathrm{H}$ $\pi / 2$ pulse, followed by a $1.0 \mathrm{~ms}$ ramped ${ }^{1} \mathrm{H}$ spin-lock pulse at $56 \mathrm{kHz}$ rf field strength. The experiments were performed with a $50 \mathrm{kHz}$ sweep width, a recycle delay of $5 \mathrm{~s}$, a ${ }^{1} \mathrm{H}$ decoupling level of $56 \mathrm{kHz}$, and 8192 scans. Exponential line broadening of $100 \mathrm{~Hz}$ was applied to all spectra. The chemical shifts of ${ }^{1} \mathrm{H}$ and ${ }^{13} \mathrm{C}$ were both referenced to TMS.

\section{SERS measurement}

SERS measurements were performed on a homemade Raman spectrometer with a $632.8 \mathrm{~nm}$ helium-neon excitation laser and triple-grating monochromator (SpectraPro 300i, Action Research). The laser beam was focused onto the sample through a Mitutoyo M Plan Apo $50 \times$ objective with 0.42 N.A. Measuring power at the samples was $1 \mathrm{~mW}$, and all spectra were collected in single scans with exposure times of $30 \mathrm{~s}$. Bare silk film and AuNP-silk film were immersed in $10 \mathrm{mM}$ 4-DMAP solution (ethanol) for $10 \mathrm{~min}$ and then dried in air.

\section{Results and discussion}

Citrate-capped AuNPs of various sizes were synthesized by a slightly modified Turkevich method, in which citrate served as both the reducing agent and the capping ligand. ${ }^{34-36}$ Increasing the amount of added citrate reduced the final size of the AuNPs. For ease of visualization, Fig. 1(A) shows a simple diagram of a single citrate-capped gold nanoparticle. The TEM images of the AuNPs (Fig. 2) indicated that the AuNPs were very monodisperse.

AuNPs were incorporated into silk by mixing aqueous AuNP solution and silk-TFA solution. TFA has been shown to rapidly dissolve silk protein. ${ }^{37}$ Dry, degummed silk was first dissolved in a small amount of TFA and then the solution was quickly injected into the aqueous AuNP solution. After stirring for over three hours, the red or purple AuNP-doped silk nanocomposite precipitated, leaving the solution clear and colourless. The nanocomposite was then processed into thin films, one of which is schematically illustrated in Fig. 1(B). Fig. 3 includes SEM images and pictures of nanocomposite films doped with a 
(A)

(B)
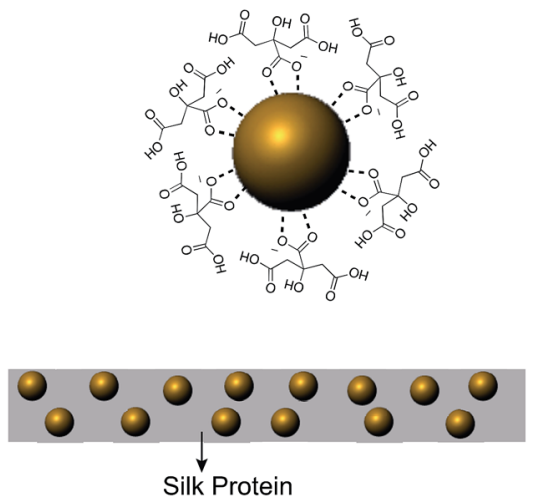

Fig. 1 (A) A single citrate-capped gold nanoparticle. (B) Schematic illustration of AuNP-doped silk film.

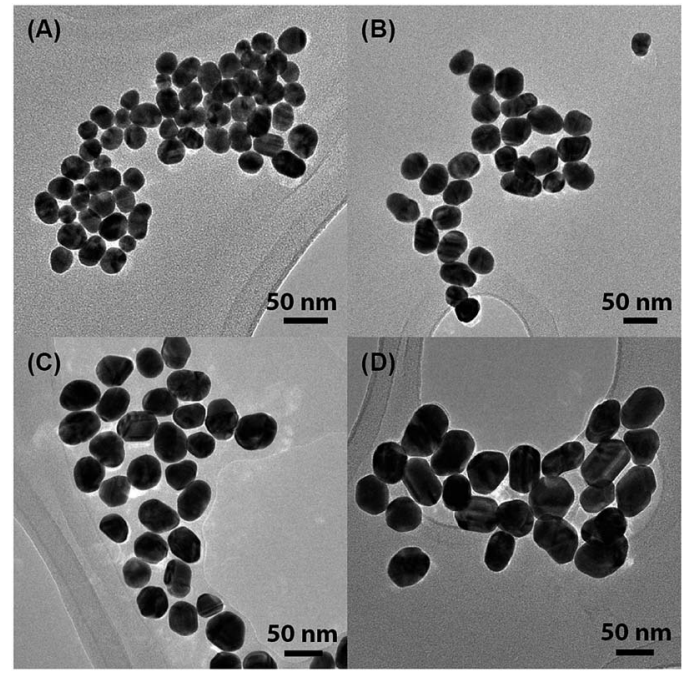

Fig. 2 TEM images of citrate-capped AuNPs of various sizes: (A) 24.4 $\pm 3.8 \mathrm{~nm}$, (B) $38.1 \pm 5.5 \mathrm{~nm}$, (C) $44.0 \pm 5.1 \mathrm{~nm}$, and (D) $50.4 \pm 5.2 \mathrm{~nm}$.

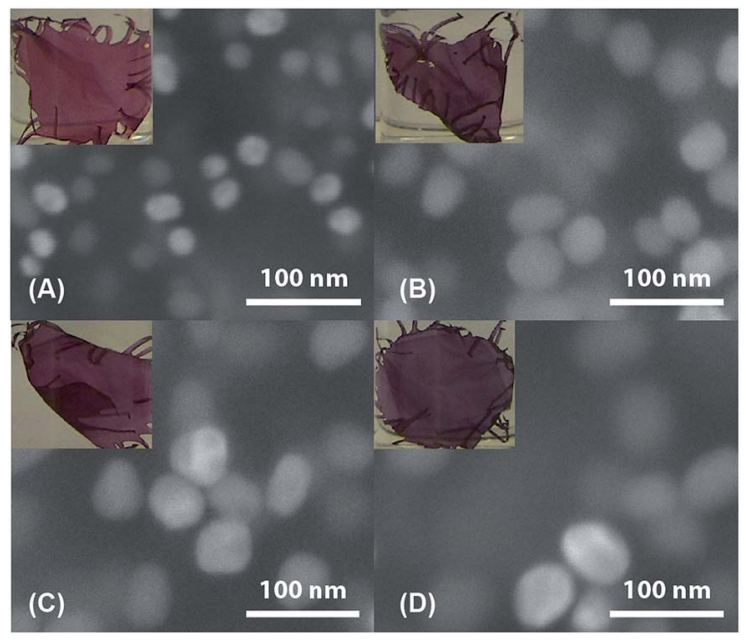

Fig. 3 High-resolution SEM images of AuNP-silk films doped with AuNPs of various sizes: (A) $24.4 \mathrm{~nm}$, (B) $38.1 \mathrm{~nm}$, (C) $44.0 \mathrm{~nm}$, and (D) $50.4 \mathrm{~nm}$. A picture of the same AuNP-silk film is shown in the corner of each image. variety of sizes of AuNPs. Comparison of these SEM images with the TEM images in Fig. 2 clearly shows that the AuNPs maintain their sizes and morphologies after being embedded in the silk, indicating that they are very stable throughout the film fabrication process. The pictures show that the colour of the AuNP-silk films changes from red to light purple as the size of the AuNPs increases. This is due to the different optical properties of differently-sized AuNPs. UV-vis spectroscopy was used to investigate these properties, which will be discussed in more detail below. It is important to note that the monodispersity of the particles in the SEM images, the homogeneous colour of the films, and the constancy of the colour of each size of AuNP (in solution and film) indicate a lack of nanoparticle aggregation in the films. Furthermore, the distribution of AuNPs in the films is likely random, and probably multilayered, as shown in Fig. 1(B).

Solid-state NMR is a powerful tool for investigating the structure of materials at the molecular level. ${ }^{38}$ In this work, we employed solid-state NMR spectroscopy to characterize degummed silkworm silk, lyophilized silkworm silk, and AuNP-silk nanocomposite. Lyophilized silkworm silk was prepared by dissolving dry, degummed silk in TFA to induce $\alpha$-helical structure before freeze-drying. ${ }^{37}{ }^{1} \mathrm{H} \rightarrow{ }^{13} \mathrm{C}$ CP-MAS NMR experiments were performed on the three samples to obtain ${ }^{13}$ C NMR spectra (Fig. 4) whose chemical shift assignments are presented in Table S1 (see ESI $\dagger$ ). Based on the NMR results, it is obvious that $\alpha$-helix is the dominant conformation in lyophilized silk ${ }^{37}$ while $\beta$-sheet is the main conformation in degummed silk and the AuNP-silk nanocomposite. Furthermore, the structure of the AuNP-silk nanocomposite is extremely similar to that of degummed silk, indicating that despite dissolution in TFA and the incorporation of nanoparticles, the nanocomposite silk largely returned to its original conformation. Structural quantification analysis of alanine $\mathrm{C}_{\beta}$ component indicates that the AuNP-silk nanocomposite has relatively less $\beta$-sheet structure than degummed silk. Some post-spinning techniques, such as immersion in ethanol or methanol, could be applied to increase the percentage of $\beta$-sheet structure.

Since AuNPs possess fascinating optical properties due to their local surface plasmon resonances, we investigated the surface plasmon absorption behaviour of AuNPs in aqueous solutions and in silk films. The results are presented in Fig. 5 and Table S2 (see ESI $\dagger$ ). From the experimental absorption spectra, several conclusions can be made: first, the peak SPR wavelength $\left(\lambda_{\mathrm{SPR}}\right)$ of AuNPs increases as the size of those AuNPs increases in both aqueous solutions and films. Second, compared with the $\lambda_{\text {SPR }}$ of AuNPs in aqueous solution, the $\lambda_{\text {SPR }}$ of AuNPs of the same size in silk films is red shifted by about $20 \mathrm{~nm}$. Third, comparison of the width of the SPR peaks clearly shows that the peaks of the AuNPs in the films are broader than those of the AuNPs in the aqueous solutions. Both the shifts and the broadening effect are due to the influence of silk protein on the AuNPs. Theoretical simulations based on Mie theory were further conducted to help explain the experimental findings and understand the influence of the silk protein on the optical properties of the 


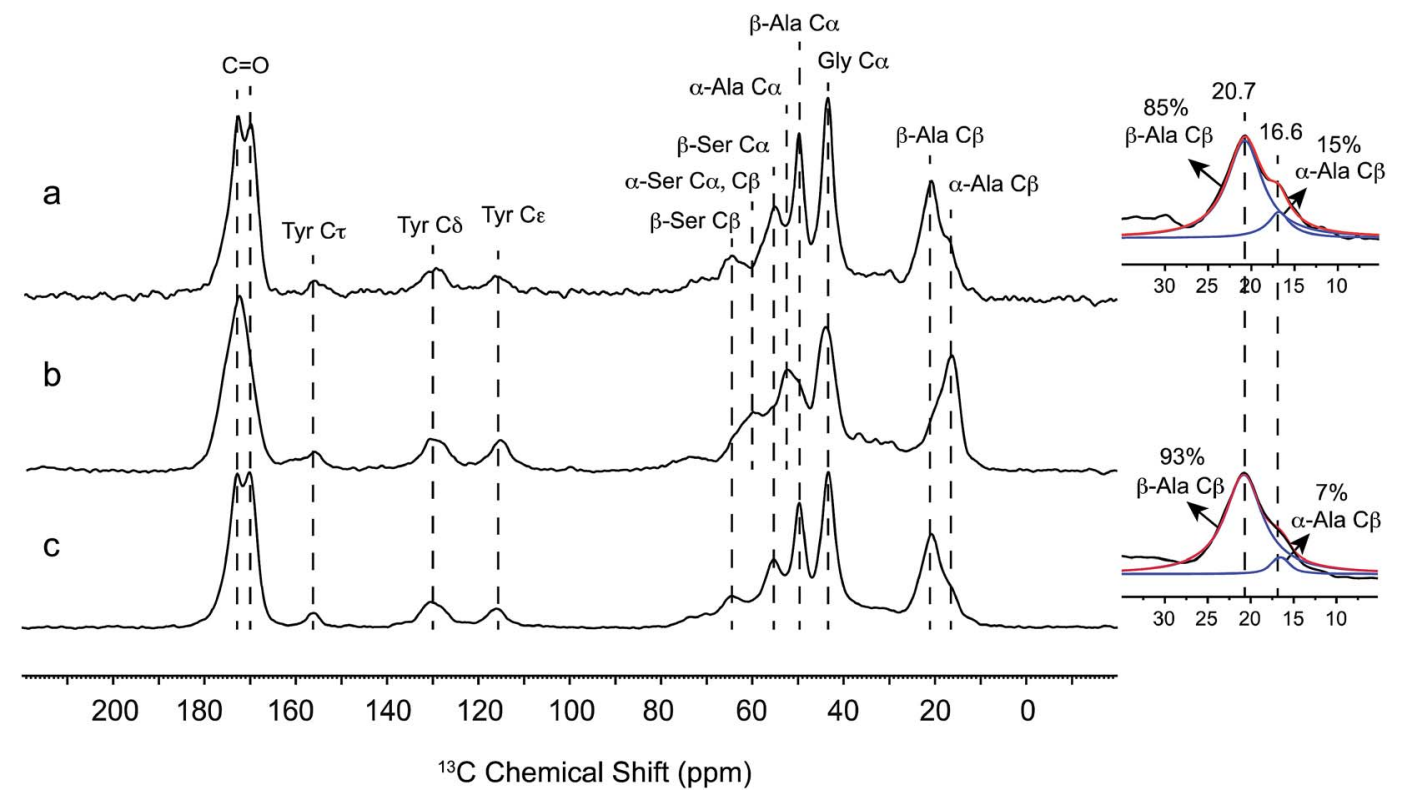

Fig. $4{ }^{1} \mathrm{H} \rightarrow{ }^{13} \mathrm{C}$ cross-polarization magic angle spinning (CP-MAS) spectra of (a) AuNP-silk nanocomposite made with $38.1 \mathrm{~nm}$ AuNPs, (b) lyophilized silk, and (c) degummed silk. All spectra were collected under $400 \mathrm{MHz}$ magnetic field with $10 \mathrm{kHz}$ spinning speed, 1 ms contact time, 5 $\mathrm{s}$ delay time, $55 \mathrm{kHz}{ }^{1} \mathrm{H}$ decoupling power and 8192 scans. Exponential line broadening of $100 \mathrm{~Hz}$ was applied on all spectra.

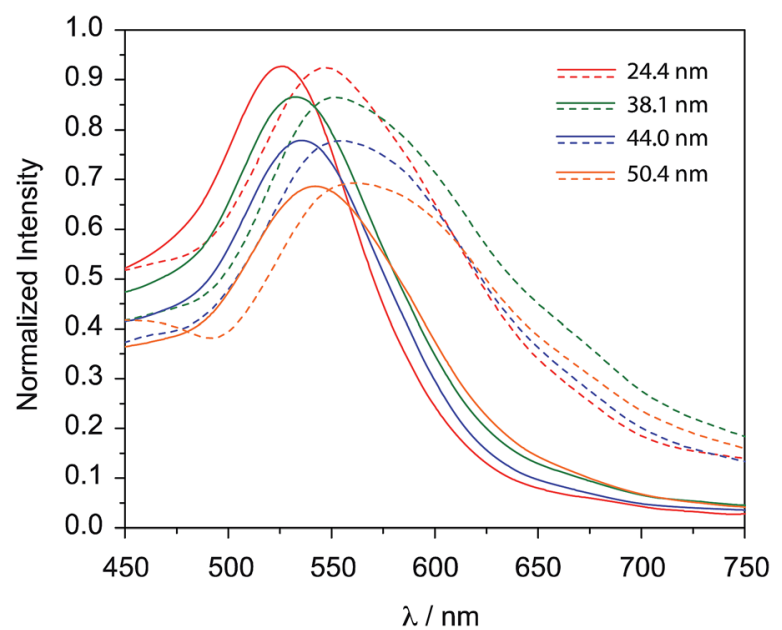

Fig. 5 Surface plasmon absorption spectra of gold nanoparticles of different diameters in aqueous solution (solid lines) and silk films (dotted lines).

AuNPs. ${ }^{39,40}$ The simulated absorption spectra are presented in Fig. 6 and the simulated results are summarized in Table S2. $\dagger$ Comparison of the simulated and experimental results shows that the $\lambda_{\text {SPR }}$ of $24.4 \mathrm{~nm}$ AuNPs is well predicted by the simulation. However, as the size of the AuNPs increases, the calculated spectra become less consistent with the experimental data (Fig. S1†). This is due to the larger size distribution and less uniform morphology of the larger AuNPs, since the simulation considers only perfectly spherical AuNPs with no size variation. Compared to the experimental $\lambda_{\text {SPR }}$ values for AuNPs in aqueous solutions, the $\lambda_{\mathrm{SPR}} \mathrm{S}$ of AuNPs in the films are red shifted by about $20 \mathrm{~nm}$. This is probably due to the difference in the refractive indices of the surrounding media, since silk protein has a larger refractive index than water. ${ }^{\mathbf{4 1 , 4 2}}$ To prove this explanation, the absorption spectra of AuNPs in silk were simulated by changing the medium refractive index to that of silk. These results (Fig. 6(C)) are highly consistent with the experimental findings. Furthermore, the broadening effect of the silk is apparent in the simulated spectra, especially for the AuNPs with larger diameters, which is also in good agreement with the experimental studies.

The prepared AuNP-silk films can be used as biocompatible SERS substrates for trace detection. A schematic representation is shown in Fig. 7. In this work, 4-DMAP and rhodamine 6G (see ESI, Fig. S2 $\dagger$ ) were used as probe molecules in SERS analysis. 4-DMAP was chosen because of its strong affinity for gold nanoparticle surfaces and distinct Raman features. One AuNP-silk film doped with $38.1 \mathrm{~nm}$ AuNPs was immersed in 10 mM 4-DMAP solution (ethanol) for $10 \mathrm{~min}$ and then dried in the air. The dry film was analysed by Raman spectroscopy. Its SERS spectrum is shown in Fig. 8(d) and the peak assignments are presented in Table $\mathrm{S} 3 . \dagger$ Five strong bands are identified in the spectra: $\mathrm{C}-\mathrm{N}-\mathrm{C}$ wagging at $759 \mathrm{~cm}^{-1}$, ring breathing and methyl rocking at $950 \mathrm{~cm}^{-1}$, trigonal bending and $\mathrm{C}-\mathrm{H}$ out-ofplane bending at $1013 \mathrm{~cm}^{-1}$, methyl rocking at $1065 \mathrm{~cm}^{-1}$, and $\mathrm{C}-\mathrm{H}$ in-plane bending at $1232 \mathrm{~cm}^{-1}$. The potential of AuNPsilk films as SERS substrates is demonstrated by the considerable enhancement in the intensities of these peaks. The calculated enhancement factor $(\mathrm{EF})$ is $\sim 150$, which is in agreement with the predicted $\mathrm{EF}$ for gold nanoparticle monomer. ${ }^{43}$ This enhancement, in combination with the fact 


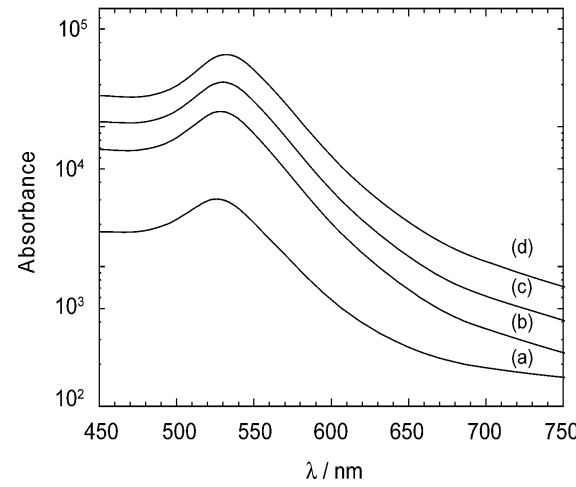

(A)

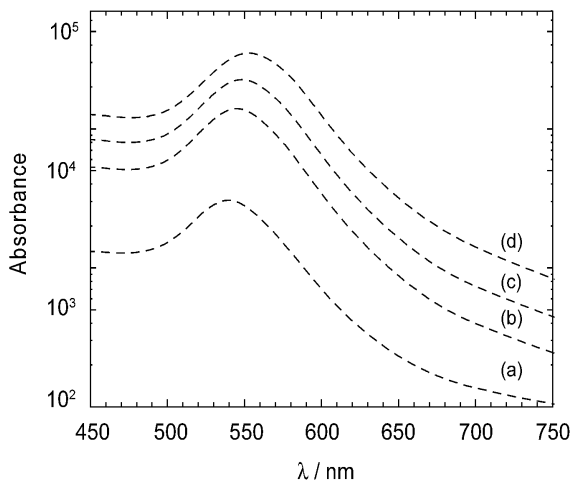

(B)

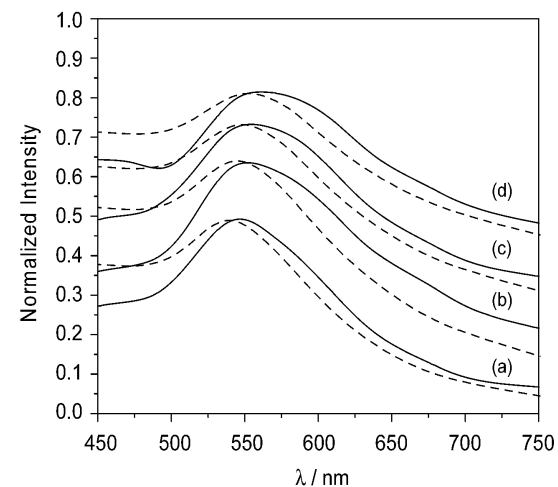

(C)

Fig. 6 Simulated surface plasmon absorption spectra for AuNPs in (A) aqueous solutions and (B) silk films with diameters of (a) $24.4 \mathrm{~nm}$, (b) 38.1 $\mathrm{nm}$, (c) $44.0 \mathrm{~nm}$, and (d) $50.4 \mathrm{~nm}$. In (C), experimental (solid lines) and simulated (dashed lines) spectra of AuNP-silk films are shown.

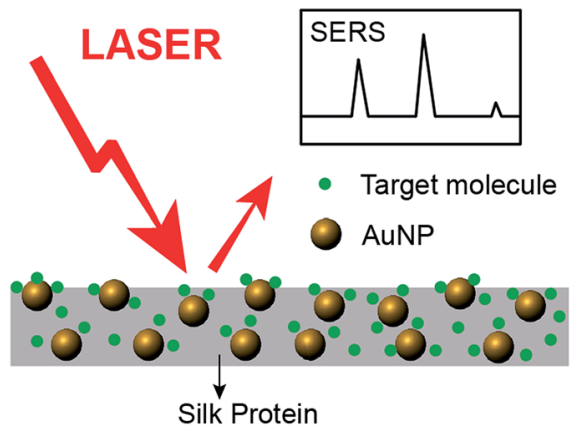

Fig. 7 Schematic representation of one AuNP-silk film used as a SERS substrate.

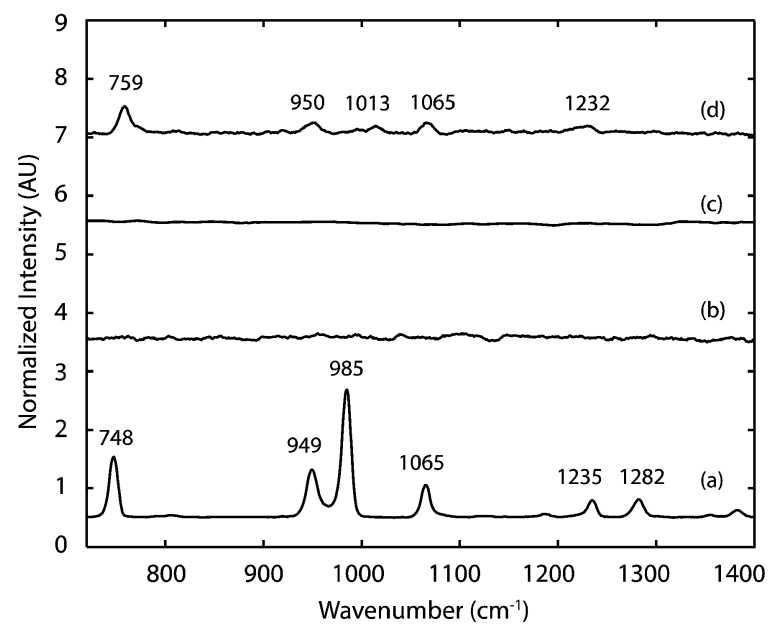

Fig. 8 Raman spectra of (a) bulk 4-DMAP, (b) bare silk film, and (c) AuNP-silk film. (d) SERS spectrum of 4-DMAP adsorbed on AuNP-silk film. The AuNP-silk film was immersed in $10 \mathrm{mM}$ 4-DMAP solution (ethanol) for $10 \mathrm{~min}$ and then dried in air.

that the AuNP-silk film is a silk-based material, makes this film a promising biocompatible material for use in in vivo biosensors.

\section{Conclusions}

In conclusion, we have described a new, rapid method to prepare AuNP-silk nanocomposite films. The AuNP-silk films were characterized by SEM, solid-state NMR spectroscopy, and UV-visible spectroscopy. The results showed good control over the size of the AuNPs embedded in the films. The NMR results demonstrated that the structure of the prepared films is very similar to that of natural silkworm silk, indicating that the fabrication procedure has a small net effect on the structure of the silk proteins. The optical properties of the AuNPs in the silk films were studied experimentally and theoretically. Experimental results show that there is a red shift of about $20 \mathrm{~nm}$ for the $\lambda_{\text {SPR }}$ of AuNPs in silk films compared with that of AuNPs in aqueous solutions, indicating that the silk changes the optical properties of the AuNPs. Simulations conducted in this work further demonstrated that the silk protein, which has a higher refractive index than water, introduces an experimentally observable shift in the absorption spectra of the AuNPs. Therefore, the optical properties of AuNPs can be tuned by incorporating them into biomaterials like silks.

As an example application of the AuNP-silk films, we have also shown in this work that they can be used as SERS substrates for high-sensitivity molecular sensing and trace analysis. The enhancement factor is not high, which is probably due to the monodispersity of the gold nanoparticles in the films. To increase the enhancement factor, we may try to incorporate more complicated structures, such as gold nanorods or gold nanoparticle dimers or trimers, into silk in the future. ${ }^{43-45}$ Such structures may create more areas of stronger EM field enhancement, or "hot spots", which are critical to the efficacy of SERS. In summary, we developed a new method for the preparation of gold nanostructure-doped silk-based biocompatible nanocomposite materials, which can be used for the development of in vivo biosensors.

\section{Acknowledgements}

This work was supported by grants from the National Science Foundation (CHE-1011937, DMR-1264801), and grants from the 
Department of Defense Air Force Office of Scientific Research (AFOSR) (FA9550-14-1-0014). We thank Dr Brian Cherry for help with NMR instrumentation and Dr Emmanuel Soignard for help with Raman instrumentation.

\section{Notes and references}

1 R. Wilson, Chem. Soc. Rev., 2008, 2028-2045.

2 J. N. Anker, W. P. Hall, O. Lyandres, N. C. Shah, Z. Jing and R. P. Van Duyne, Nat. Mater., 2008, 7, 1-12.

3 J. Nam, N. Won, H. Jin, H. Chung and S. Kim, J. Am. Chem. Soc., 2009, 131, 13639-13645.

4 Z. Q. Tian, B. Ren and D.-Y. Wu, J. Phys. Chem. B, 2002, 106, 9463-9483.

5 B. Sharma, R. R. Frontiera, A.-I. Henry, E. Ringe and R. P. Van Duyne, Mater. Today, 2012, 15, 16-25.

6 D. Kaplan, W. W. Adams and B. Farmer, Silk: biology, structure, properties, and genetics, ACS symposium series, 1994.

7 Z. Shao and F. Vollrath, Nature, 2002, 1.

8 G. H. Altman, F. Diaz, C. Jakuba, T. Calabro, R. L. Horan, J. Chen, H. Lu, J. Richmond and D. L. Kaplan, Biomaterials, 2002, 1-16.

9 D. N. Rockwood, R. C. Preda, T. Y. U. cel, X. Wang, M. L. Lovett and D. L. Kaplan, Nat. Protoc., 2011, 6, 16121631.

10 H. Tao, D. L. Kaplan and F. G. Omenetto, Adv. Mater., 2012, 24, 2824-2837.

11 K. J. Koski, P. Akhenblit, K. McKiernan and J. L. Yarger, Nat. Mater., 2013, 12, 262-267.

12 S. Kim, A. N. Mitropoulos, J. D. Spitzberg, H. Tao, D. L. Kaplan and F. G. Omenetto, Nat. Photonics, 2012, 1-6. 13 A. Singh, S. Hede and M. Sastry, Small, 2007, 3, 466-473.

14 H. Tao, S. M. Siebert, M. A. Brenckle, R. D. Averitt, M. CroninGolomb, D. L. Kaplan and F. G. Omenetto, Appl. Phys. Lett., 2010, 97, 123702.

15 T. Cohen-Karni, K. J. Jeong, J. H. Tsui, G. Reznor, M. Mustata, M. Wanunu, A. Graham, C. Marks, D. C. Bell, R. Langer and D. S. Kohane, Nano Lett., 2012, 12, 5403-5406.

16 E. Kharlampieva, V. Kozlovskaya, B. Wallet, V. V. Shevchenko, R. R. Naik, R. Vaia, D. L. Kaplan and V. V. Tsukruk, ACS Nano, 2010, 4, 7053-7063.

17 C. W. P. Foo, S. V. Patwardhan, D. J. Belton, B. Kitchel, D. Anastasiades, J. Huang, R. R. Naik, C. C. Perry and D. L. Kaplan, Proc. Natl. Acad. Sci. U. S. A., 2006, 1-6.

18 E. Kharlampieva, V. Kozlovskaya, R. Gunawidjaja, V. V. Shevchenko, R. Vaia, R. R. Naik, D. L. Kaplan and V. V. Tsukruk, Adv. Funct. Mater., 2010, 20, 840-846.

19 J. Ayutsede, M. Gandhi, S. Sukigara, H. Ye, C.-M. Hsu, Y. Gogotsi and F. Ko, Biomacromolecules, 2006, 7, 208-214.

20 H. Pan, Y. Zhang, Y. Hang, H. Shao, X. Hu, Y. Xu and C. Feng, Biomacromolecules, 2012, 13, 2859-2867.
21 E. Steven, W. R. Saleh, V. Lebedev, S. F. A. Acquah, V. Laukhin, R. G. Alamo and J. S. Brooks, Nat. Commun., 2013, 4, 1-8.

$22 \mathrm{~K} . \mathrm{Hu}$, M. K. Gupta, D. D. Kulkarni and V. V. Tsukruk, Adv. Mater., 2013, 25, 2301-2307.

23 S. Nie and S. R. Emory, Science, 1997, 275, 1102-1106.

24 P. L. Stiles, J. A. Dieringer, N. C. Shah and R. P. Van Duyne, Annu. Rev. Anal. Chem., 2008, 1, 601-626.

25 J. F. Li, Y. F. Huang, Y. Ding, Z. L. Yang, S. B. Li, X. S. Zhou, F. R. Fan, W. Zhang, Z. Y. Zhou, D. Yin Wu, B. Ren, Z. L. Wang and Z. Q. Tian, Nature, 2010, 464, 392-395.

26 M. Fleischmann, P. J. Hendra and A. J. McQuillan, Chem. Phys. Lett., 1974, 26, 1-4.

27 D. L. Jeanmaire and R. P. Van Duyne, J. Electroanal. Chem., 1977, 84, 1-13.

28 H. Liu, L. Zhang, X. Lang, Y. Yamaguchi, H. Iwasaki, Y. Inouye, Q. Xue and M. Chen, Sci. Rep., 2011, 1, 112.

29 X. Jiang, Z. Jiang, T. Xu, S. Su, Y. Zhong, F. Peng, Y. Su and Y. He, Anal. Chem., 2013, 85, 2809-2816.

30 J. Turkevich, P. C. Stevenson and J. Hillier, Discuss. Faraday Soc., 1951, 11, 55.

31 B. V. Enustun and J. Turkevich, J. Am. Chem. Soc., 1963, 85, 3317-3328.

32 S. R. H. A. E. L. Hahn, Phys. Rev., 1962, 128, 2042-2053.

33 B. H. Meier, Chem. Phys. Lett., 1992, 18, 201-207.

34 J. Kimling, M. Maier, B. Okenve, V. Kotaidis, H. Ballot and A. Plech, J. Phys. Chem. B, 2006, 110, 15700-15707.

35 X. Ji, X. Song, J. Li, Y. Bai, W. Yang and X. Peng, J. Am. Chem. Soc., 2007, 129, 13939-13948.

36 M. Doyen, K. Bartik and G. Bruylants, J. Colloid Interface Sci., 2013, 399, 1-5.

37 S.-W. Ha, A. E. Tonelli and S. M. Hudson, Biomacromolecules, 2005, 6, 1722-1731.

38 C. Dybowski and S. Bai, Anal. Chem., 2008, 80, 4295-4300.

39 G. Mie, Ann. Phys., 1908, 25, 377-445.

40 W. Haiss, N. T. K. Thanh, J. Aveyard and D. G. Fernig, Anal. Chem., 2007, 79, 4215-4221.

41 B. D. Lawrence, M. Cronin-Golomb, I. Georgakoudi, D. L. Kaplan and F. G. Omenetto, Biomacromolecules, 2008, 9, 1214-1220.

42 S. T. Parker, P. Domachuk, J. Amsden, J. Bressner, J. A. Lewis, D. L. Kaplan and F. G. Omenetto, Adv. Mater., 2009, 21, 24112415.

43 Z. Zhang, P. Yang, H. Xu and H. Zheng, J. Appl. Phys., 2013, 113, 033102.

44 D.-K. Lim, K.-S. Jeon, J.-H. Hwang, H. Kim, S. Kwon, Y. D. Suh and J.-M. Nam, Nat. Nanotechnol., 2011, 1-9.

45 S. L. Kleinman, R. R. Frontiera, A.-I. Henry, J. A. Dieringer and R. P. Van Duyne, Phys. Chem. Chem. Phys., 2012, 15, 21-36. 